\title{
Magnetoresistance of atomic-size contacts realized with mechanically controllable break junctions
}

\author{
Stefan Egle, ${ }^{1}$ Cecile Bacca, ${ }^{1}$ Hans-Fridtjof Pernau, ${ }^{1}$ Magdalena Huefner, ${ }^{2}$ Denise Hinzke, ${ }^{1}$ Ulrich Nowak, ${ }^{1}$ and \\ Elke Scheer ${ }^{1}$ \\ ${ }^{1}$ Department of Physics, University of Konstanz, 78457 Konstanz, Germany \\ ${ }^{2}$ Solid State Physics Laboratory, ETH Zurich, 8093 Zurich, Switzerland
}

(Received 22 October 2009; revised manuscript received 10 February 2010; published 1 April 2010)

\begin{abstract}
We present a comprehensive study of the conductance behavior of atomic-size contacts made of ferromagnetic metals $(\mathrm{Co})$ or noble metals $(\mathrm{Au})$ with ferromagnetic electrodes $(\mathrm{Co})$. In order to separate the influence of the large electrodes from the influence of the contacts themselves, we used different sample geometries. These include combinations of nonmagnetic electrodes connected to magnetic bridges and vice versa as well as different orientations of the magnetic field. The magnetoresistance (MR) curves show very rich behavior with strong MR ratios (MRR). In all geometries the MRR values are of comparable size, reaching up to a few thousand percent in the tunneling regime. We study the possible influence of the micromagnetic order of the domains in the vicinity of the contact as well as ballistic MR, giant MR, tunnel MR, atomically enhanced anisotropic MR (AAMR), and magnetostriction. We conclude that AAMR is the most important origin for the MR at high magnetic fields $(|B|>2 \mathrm{~T})$, while magnetostriction, tunnel MR, and giant MR govern the low-field regime $(|B|<2 \mathrm{~T})$.
\end{abstract}

PACS number(s): 73.23.Ad, 73.43.Qt, 73.63.Rt, 75.47.De

\section{INTRODUCTION}

For possible use of magnetic properties in electronic devices, magnetic point contacts have been investigated intensively during the last years. In particular, the possibility to dramatically change the resistance by applying a small magnetic field has been studied in detail. Nevertheless, the experimental situation is still contradictory. This holds true for the so-called conductance histograms, which are used to determine the preferred conductance values of quantum point contacts as well as the magnetoresistance (MR) ratios which in some experiments reach values of $100000 \%$. $^{1}$ The mechanism of the high MR is controversial as well. Effects due to the micromagnetic order of the domains in the vicinity of the contact, anisotropic MR (AMR), giant MR (GMR), tunnel MR (TMR), ballistic MR (BMR), ${ }^{2,3}$ magnetostriction, and further effects depending on the actual realization of the contacts are discussed. ${ }^{4}$

The conductance histograms strongly depend on the experimental realization. The results range from complete absence of preferred conductance values ${ }^{5}$ via a small number of rather broad structures ${ }^{6}$ to almost perfectly quantized values at half integers of $G_{0}$ upon application of an external field. ${ }^{7}$ The spin properties of the conducting electrons in point contacts have been addressed theoretically by several groups. ${ }^{4}$ However, only few direct experimental proofs exist. ${ }^{8,9}$ Often the appearance of preferred conductance values at half integers $G=N G_{0} / 2$ of the conductance quantum $G_{0}=2 e^{2} / h$ is interpreted as indications for complete spin polarization. ${ }^{7}$ The underlying assumption is that any spin-degenerate atomic contact should have quantized conductance in multiples of $G_{0}$ given by a number $N$ of transport channels with perfect transmission $i=1$. Calculations of the conduction channels of ferromagnetic atomic-size contacts predict strong but configuration-dependent polarization values, ${ }^{10}$ which do not yield half integer values of the total conductance. Both the number $N$ and the transmission $i$ of the transport channels differ for both spin orientations. The $i$ may acquire arbitrary values between 0 and 1 , which can be influenced by external magnetic fields. ${ }^{11}$ Therefore, the MR curves may display rich behavior. Interestingly, the size and even the sign of the MR may differ significantly upon slight variations in the atomic configuration. The MR may vanish for particular configurations. Similarly, due to atomic rearrangements, the conductance histograms, i.e., the statistical analysis of preferred conductance values, are expected to depend on the applied magnetic field if spin polarization dominates the conductance.

Herein, the possible influence of the spin polarization onto the electronic transport is investigated by analyzing the MR through atomic-size contacts in different orientations of the applied magnetic field with respect to the film plane and current direction. In order to separate the influence of the large electrodes from the influence of the contact itself, we vary the sample geometry. We argue that magnetostriction contributes to the observed MR and is not negligible. For samples consisting of two magnetic electrodes strong resistance changes are caused by TMR and GMR. We find that the continuous changes in the MR up to approximately $\pm 2 \mathrm{~T}$ are due to the reorientation of the magnetic domains in the electrodes, whereas the changes at higher fields are due to an enhancement of the saturation field in the constriction region. The constriction causes a local variation in the band structure and reduces the quenching of the orbital magnetic moment. Because of the limited number of neighboring atoms in the constriction region, the orbital character of the wave functions is not completely suppressed, but the currentcarrying electronic modes partially maintain their orbital properties. This results in a strong spin-orbit scattering in atomic-size contacts. The spin-orbit scattering may give rise to a pronounced field dependence of the resistance of the contacts. Because the local band structure is given by the overlap of the wave functions of the neighboring atoms, it is strongly dependent on the geometry on the atomic scale. 


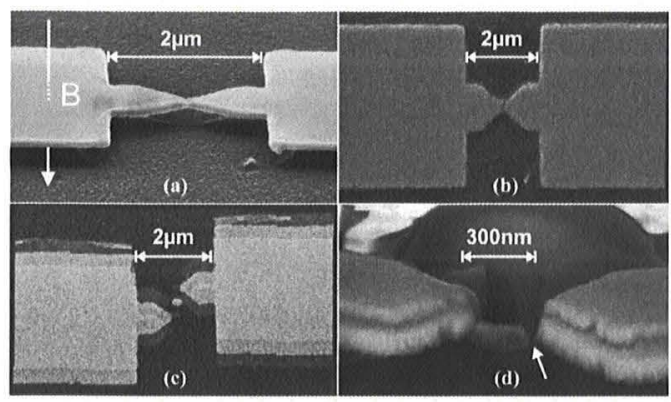

FIG. 1. Scanning electron micrographs of the different types of samples under study. (a) Inclined view of a suspended Co break junction with indication of the orientation of the magnetic field for recording the MR. (b) Top view of a nonsuspended Co bridge after measurement. The remaining break is clearly visible. (c) Top view of a Co-Au-Co sample fabricated by three-angle shadow evaporation. The $\mathrm{Au}$ areas appear in light gray (although partially covered by the Co layers), the Co areas are dark gray. (d) Inclined view of a $\mathrm{Au}-\mathrm{Co}-\mathrm{Au}$ sample fabricated by two-angle shadow evaporation after dismounting from the cryostat with the break visible at the right interface (arrow). Here the lower part of the metal film consists of $\mathrm{Au}$, the upper parts, and the nanobridge of $\mathrm{Co}$.

These effects have been called atomically enhanced AMR (AAMR). They should not be confused with the BMR effect. Also BMR considers local changes of the band structure at the constriction. However, it assumes only fully transmitted conductance channels, the number of which changes upon application of a magnetic field. In particular, the number of modes for spin-up and spin-down bands may differ from each other. Thus, in the BMR picture only multiples of the spin-split conductance quantum $e^{2} / h$ are possible while in the AAMR picture arbitrary values of the conductance occur. To summarize, AAMR affects both, the saturation field as well as conductance of the contacts. Finally, the observed abrupt resistance jumps can partially be attributed to atomic rearrangements triggered by magnetostrictive forces.

The paper is organized as follows: Sec. II describes the sample preparation and briefly describes the experimental setups. Section III is divided into three parts, each one being dedicated to the presentation of the transport measurements conducted on the three different sample types under investigation. The analysis and interpretation of our results is given in Sec. IV. This includes a Sec. IV A which reports micromagnetic simulations of the domain structure of the nanobridges with or without break. We discuss the influence of magnetostriction in Sec. IV B, the origins of hysteretic MR in Sec. IV C. Finally, the findings are summarized in Sec. V.

\section{EXPERIMENTAL}

We investigate pure cobalt samples [see Figs. 1(a) and 1(b)] with a symmetric and an asymmetric layout. We also study a combination of nonmagnetic gold electrodes with magnetic bridges and vice versa. These samples consist of a bridge of about a few hundred nanometers in length connected to two micrometer-sized leads, consisting of another material than the bridge itself [see Figs. 1(c) and 1(d)].
TABLE I. Overview of the different sample types and the MRR of the samples in the atomic contact regime and tunnel regime. The triangular-shaped inner part of the structure can be symmetric (sym) or asymmetric (asym), as well as suspended (u) or nonsuspended (nu).

\begin{tabular}{ccccc}
\hline \hline No. & Materials & Geometry & $\begin{array}{c}\text { MRR atomic } \\
(\%)\end{array}$ & $\begin{array}{c}\text { MRR tunnel } \\
(\%)\end{array}$ \\
\hline 1 & Co & Sym/u & $9-70$ & $100-220$ \\
2 & Co & Asym/u & $6-55$ & 520 \\
3 & Co & Sym/nu & $15-75$ & $200-460$ \\
4 & Co & Asym/nu & $17-60$ & $150-620$ \\
5 & Co-Au-Co & Sym/u & $21-100$ & $7700-14000$ \\
6 & Au-Co-Au & Sym/u & $1-33$ & $600-1470$ \\
\hline \hline
\end{tabular}

We used bronze substrates covered with a micrometerthick polyimide layer which provides electrical isolation of the substrate from our sample structures. The latter are fabricated via e-beam lithography using a double-layer system of a polymethyl methacrylate and a copolymer positive resist. The different materials are deposited subsequently without breaking the vacuum of $p \leq 10^{-3} \mathrm{~Pa}$ by using shadow evaporation through a suspended mask. This process enables good electric contact between the metals. For the samples consisting of two metals, we first evaporate the Au layer followed by the Co layers. The thickness of both the Co and the Au films is $100 \mathrm{~nm}$, unless stated otherwise. As an artifact of the shadow evaporation technique, the electrodes at a distance of more than a few hundred nanometers consist of bilayers of $\mathrm{Co}$ and $\mathrm{Au}$. The separation between the parasitic Co layers and the Co nanobridge is larger than the spindiffusion length in $\mathrm{Au},{ }^{12} \lambda_{s f} \approx 100 \mathrm{~nm}$. This means that for the Au-Co-Au samples a possible stray-field contribution of the parasitic Co electrodes has to be considered. However, since the voltage drops locally at the atomic contact, this does not affect the resistance directly. Finally, the triangularshaped central part can be suspended by means of etching the polyimide layer in a reactive oxygen plasma, as shown in Fig. 1(a). Herein, we report data taken on six samples. An overview of these samples is given in Table I.

The size and resistance of the contacts are adjusted using the mechanically controllable break-junction technique. ${ }^{13,14}$ A three-point bending mechanism and a motor with welldefined gear reduction allows us to open the contact in a controlled way while the sample is always kept at temperatures below $4 \mathrm{~K}$ in cryogenic vacuum. No contamination of the samples was detected as long as the samples were kept under these conditions. When raising the temperature to more than $\approx 10 \mathrm{~K}$, gas atoms start to accumulate at the surface. They impede the fabrication of clean metallic atomic contacts. The cryostats are furthermore equipped with superconducting solenoids in order to record the MR in perpendicular magnetic field up to $8 \mathrm{~T}$. Part of the measurements have been performed in a vector magnet with fields of up to $0.5 \mathrm{~T}$ in each direction. The resistance is measured with various electronic setups by applying bias voltages below $2 \mathrm{mV}$ in order to avoid self-heating and to ensure linear transport conditions. The measurements are performed in a mesos- 

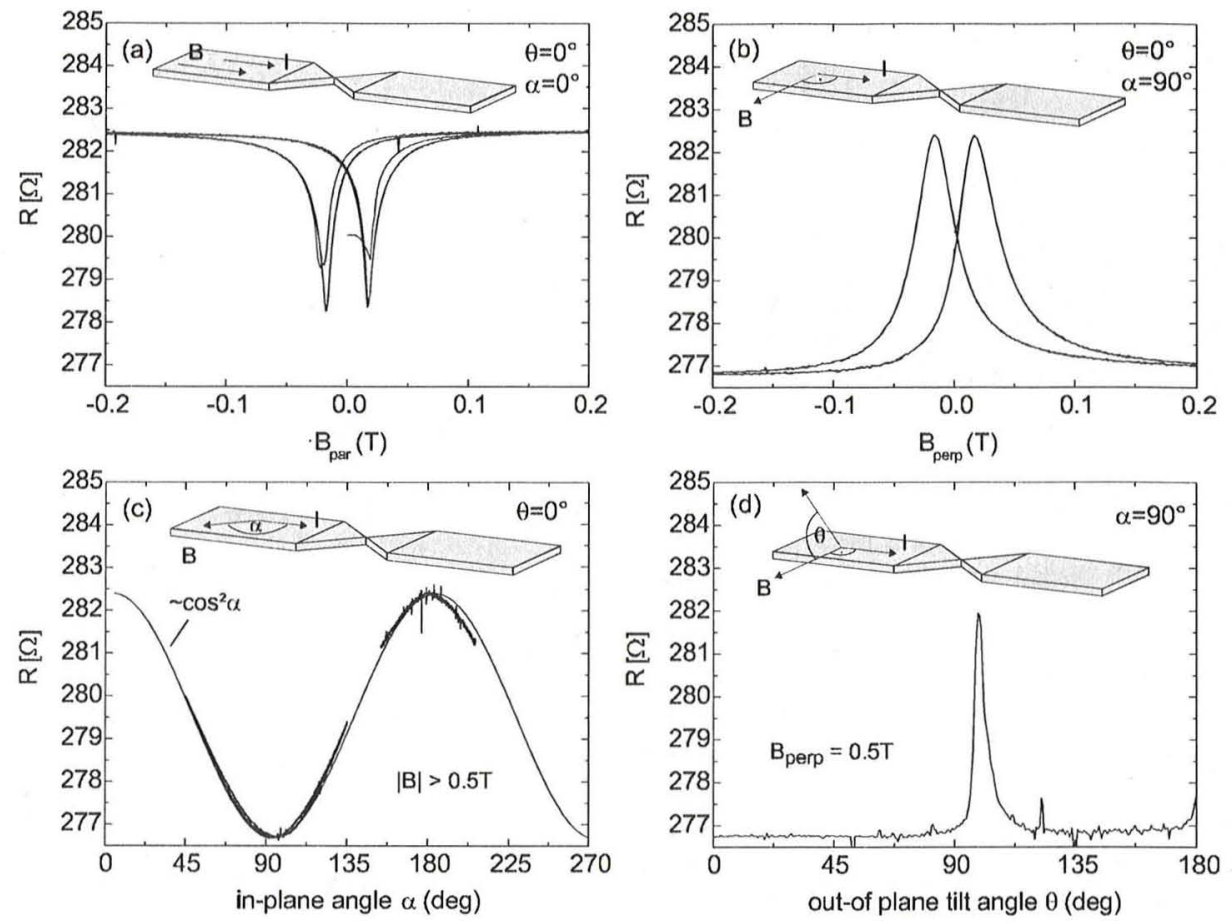

FIG. 2. Magnetoresistance of a nonsuspended and unbroken Co nanobridge. The field has been swept repeatedly in the direction indicated by the arrows. (a) B in plane and parallel to I. (b) B in plane but applied perpendicularly to $\mathbf{I}$. (c) Resistance as a function of the azimuthal angle for a field of $0.5 \mathrm{~T}$. The solid line is a fit to the expected $\cos ^{2} \alpha$ dependence. (d) Resistance as a function of the inclination of the field of $B=0.5 \mathrm{~T}$ out of plane.

copic two-point configuration. Current and voltage leads are separated from each other at the sample holder. This means that the indicated resistance values include small and lead resistances in the order of a few ohms. Upon permanently opening and closing the bridge several hundred times, we record the linear conductance from which we calculate conductance histograms. We verified that the current-voltage characteristics are linear in this regime with a precision of roughly $1 \%$ and that the shape of the opening traces and conductance histograms to not depend on the bias voltage.

\section{RESULTS}

\section{A. Cobalt samples}

In order to determine the influence of the usual AMR of the Co film, we first investigate the angular dependence of the MR of an unbroken and nonsuspended nanobridge (thickness $30 \mathrm{~nm}$ ) with a total resistance of approximately $280 \Omega$ at $4 \mathrm{~K}$. A magnetic field of $500 \mathrm{mT}$ is applied parallel to the film plane. Figures 2(a) and 2(b) demonstrate the typical AMR behavior which has been observed for nanowires realized by various techniques. ${ }^{15-18}$

As known for AMR and reported earlier for nanoconstrictions of $\mathrm{Fe}$ and $\mathrm{Ni},{ }^{19}$ the resistance is highest when the field is applied parallel to the current and smallest for perpendicular orientation. Figure 2(c) depicts the resistance as a function of the angle between the current direction and the field. The shape follows the common $\cos ^{2} \alpha$ dependence expected for AMR in saturated field with an amplitude of approximately $2 \%$ of the total resistance. In Fig. 2(d) we show the change in the resistance when turning a field of size $B$ $=0.5 \mathrm{~T}$ out of plane for perpendicular orientation to the current. The resistance remains at its low level up to about $\theta$ $=90^{\circ}$, then reaches a maximum for $\theta=100^{\circ}$ and relaxes back to the low level when further increasing the angle. The experiment demonstrates that the magnetization direction is preferably in plane. For a field of $0.5 \mathrm{~T}$ and in the narrow angular range around $100^{\circ}$, part of the magnetization turns out of plane. The fact that the maximum is not observed at $\theta=90^{\circ}$ is due to an uncertainty of our angle determination. In summary, these measurements underline that the behavior of the unbroken and nonsuspended bridge follows the expected AMR behavior reported earlier. ${ }^{20}$

Also, when applying the field perpendicular to the film the MR of the unbroken samples is less than $3 \%$ up to field amplitudes of $5 \mathrm{~T}$ for all samples investigated. For the CoAu-Co samples and Au-Co-Au samples it is less than $2 \%$. Before break the samples with a metal thickness of $\approx 100 \mathrm{~nm}$ have a resistance of typically $10-15 \Omega$ at low temperature. The MR is negative, i.e., the resistance decreases with increasing field amplitude. The functional shape of this decrease is bell shaped for the Co-only samples and the Co-Au-Co samples. No sharp feature can be detected at $1.8 \mathrm{~T}$. However, the field dependence in general becomes weaker above roughly $2 \mathrm{~T}$, as found for thin Co films. ${ }^{21}$ The $\mathrm{MR}$ of the Au-Co-Au samples decreases linearly up to $5 \mathrm{~T}$. For comparison we also investigated Au-only samples. They 


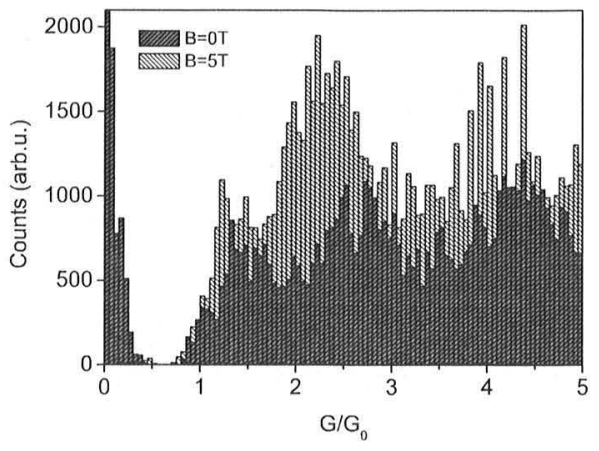

FIG. 3. Conductance histograms of a Co break junction measured at $T=1.6 \mathrm{~K}$ in zero field (right shaded, black bars) and perpendicular field of $5 \mathrm{~T}$ (left shaded, white bars). Broad maxima are observed at $1.3 G_{0}$ and at $2.5 G_{0}$.

showed very small MR (smaller than $1 \%$ ) in the same field range but a slight increase rather than a decrease as expected for normal metals by the conventional MR due to the Lorentz force.

Addressing now the magnetotransport of the atomic-size contacts arranged with these nanobridges, our first aim is to determine the preferred conductance values, because these values are characteristic for each material. In many experiments the preferred conductance values of single-atom contacts of gold have been determined to be $G=1 G_{0}$ with very good precision. ${ }^{22}$ In our measurements the conductance histogram of Co reveals a first broad maximum around $1.3 G_{0}$ and a second one around $2.5 G_{0}$, both in zero field and in an applied perpendicular field of $5 \mathrm{~T}$ (Fig. 3).

The exact shape, position, and height of the maxima vary from sample to sample. As shown below, this is related to the variation in the remanent magnetization state of the bridges, which gives rise to resistance variations in the atomic-sized contacts. Comparing the histograms recorded on various samples we conclude that neither the substructure of the main peaks is significant nor is the small shift of the second maximum to slightly lower conductance values. Our findings are in accordance with an experiment in which break junctions made of notched wires (instead of thin evaporated films) and an in-plane field orientation were used. ${ }^{6}$

The absence of a splitting or shift of the maxima is an indication that the preferred conductance values are not caused by the BMR effect. The good agreement between the histograms recorded with and without magnetic field is all the more surprising when considering the magnetoresistance traces of atomic-size Co contacts. The MR traces of the Co samples show a broad variation in possible shapes. In particular, rearrangements of contacts of the same sample may depict very distinct shapes. Resistance minima as well as maxima at zero field are possible for the same sample. A subset of typical MR curves of two samples is depicted in Fig. 4. In between the measurements of the MR traces of the individual contacts the bridges have been closed completely and reopened repeatedly in order to prepare a different atomic configuration for each contact. In the literature, various definitions of the MR ratio are used. Since in our samples neither the resistance in antiparallel or parallel ori-
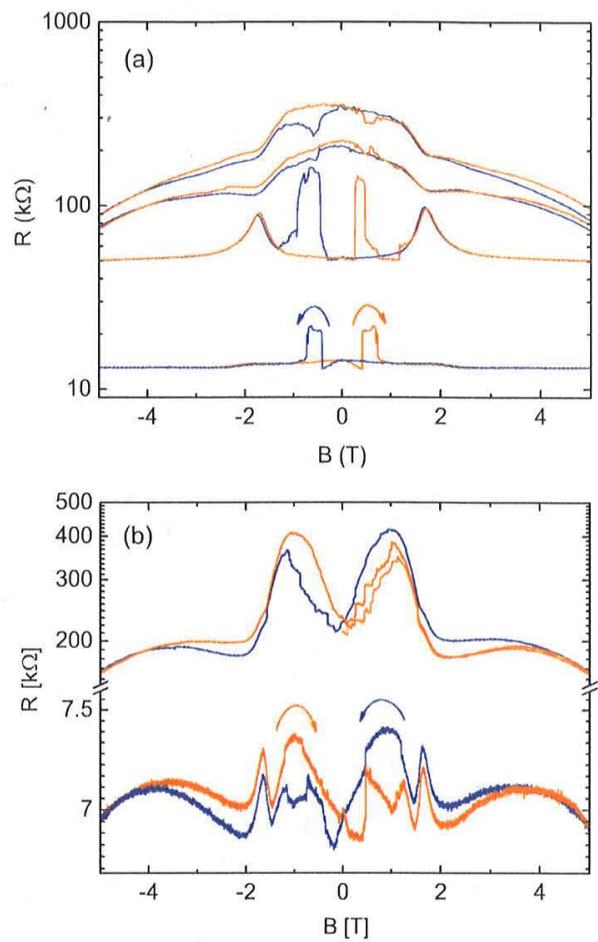

FIG. 4. (Color online) MR measurements of two different Co break junctions of type No. 1 in the single-atom and tunnel regime for $T \sim 2 \mathrm{~K}$ (magnetic field applied perpendicular to sample plane). The gray (online: orange) curves represent a sweep direction from left to right, the black (online: blue) ones the opposite way. The shape is characterized by a hysteretic structure for $0<|B|<1$ T and a reversible structure for larger fields with a dip, a peak or a kink at $\pm 1.8 \mathrm{~T}$. In many atomic contacts, large hysteretic resistance jumps occur upon raising the field size.

entation can be defined, nor the resistance at zero field adopt an extremal value, and in addition a saturation at maximum field is not achieved, we choose the following definition:

$$
\mathrm{MRR}=\left(R_{\max }-R_{\min }\right) / R_{\min } .
$$

The MR ratios (MRR) according to this definition amounts to $70 \%$ in the few-atom regime and can achieve several hundred percent in the tunneling regime. In the contact regime we observe hysteretic as well as nonhysteric structures. Although the shapes of the MR traces are manifold, several robust observations can be noted: in the low-field regime $(|B|<1.5 \mathrm{~T})$, a stepwise change in the resistance can occur. At higher fields, the resistance change is continuous and seems to saturate. The discontinuous behavior is mostly absent for tunnel contacts, only in some examples a TMR-like switching behavior is observed at low fields. Nevertheless, the majority of features is present in both sweep directions. In all traces, an additional feature appearing for both polarities is observed near the saturation field of Co at $|B|$ $=1.8 \mathrm{~T}$. This can be a resistance maximum as well as a minimum or a shoulder.

In order to deduce the influence of anisotropies of the electrodes, which may give rise to different switching fields 

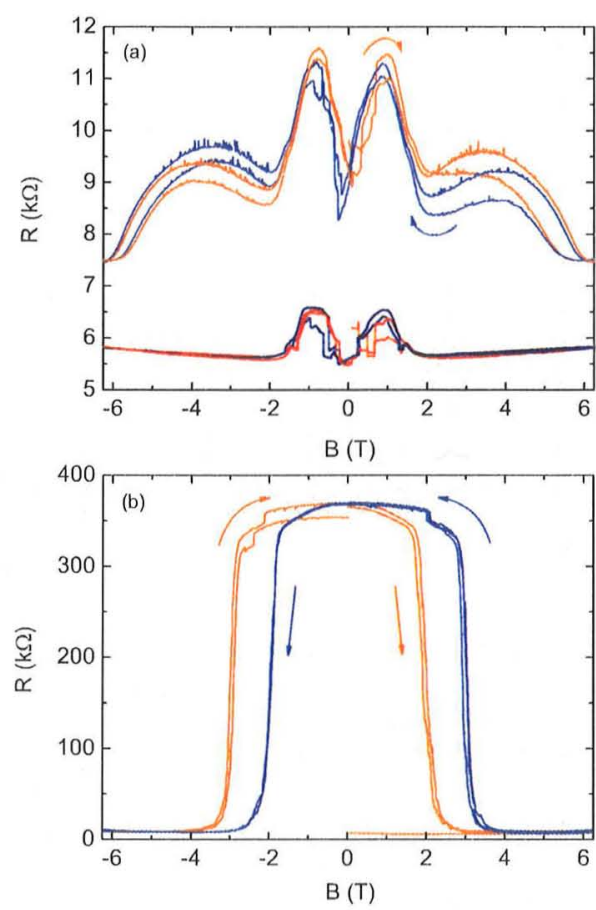

FIG. 5. (Color online) MR traces of contacts realized with a suspended Co nanobridge with asymmetric electrodes (sample No. 2) measured at $1.5 \mathrm{~K}$ in perpendicular field. One of the electrodes has a width of $0.5 \mu \mathrm{m}$, the other one $1 \mu \mathrm{m}$. The sample has been demagnetized and thoroughly closed between the measurements shown in panels (a) and (b), respectively.

of the magnetization, we also produced samples with asymmetric shape of the electrodes on the micrometer scale. In most cases, the amplitude of the magnetoresistance effects did not change significantly. However, traces with high resistance at zero field and hysteresis between sweeping up and down the field appeared more often while traces with multiple maxima are observed less frequently. Examples for both types are shown in Fig. 5. Magnetostrictive effects on the MR were studied by varying the length of the suspended part and by removing the suspension completely. Because of the thickness of the samples of roughly $100 \mathrm{~nm}$ magnetostriction cannot be excluded even for the nonsuspended samples. However, we expect the contribution of magnetostriction to the MR to be much smaller for these samples. Interestingly, the resulting MR traces show a similar complexity and the MRR values were of the same order of magnitude (see Fig. 6). From these experiments we conclude that the field dependence of the conduction channels themselves causes the rich effects. Since the GMR and TMR-like features persist in all samples, we furthermore conclude that the orientation of the magnetization of the electrodes are important for the overall shape of the MR curves (maximum resistance at low field, minimum resistance at high field).

\section{B. Co-Au-Co}

In order to separate the influence of the micromagnetic order from the atomistic effects we fabricated samples con-

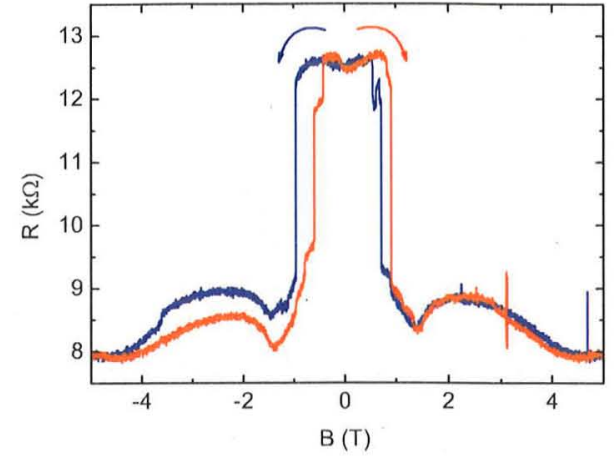

FIG. 6. (Color online) MR traces recorded on contacts of an nonsuspended Co bridge (sample No. 3) measured at $T=1.5 \mathrm{~K}$ in perpendicular field.

sisting of a nonmagnetic central part, in which the atomic contact was supposed to form, and magnetic electrodes. The conductance histogram (not shown) shows features at $1 G_{0}$ (which would be expected for gold contacts) as well as at the values which are typical for Co. Individual opening traces reveal that either plateaus at $1 G_{0}$ or at $1.3 G_{0}$ are observed. From these findings we deduce that the rupture occurred close to one of the interfaces between $\mathrm{Co}$ and $\mathrm{Au}$ [see Fig. 1(c)], which gives rise to either $\mathrm{Au}-\mathrm{Au}$ or $\mathrm{Co}-\mathrm{Co}$ contacts. Au-Co contacts may form as well, however, since their preferred conductance values are not known, we cannot identify them in the histogram.

The MR traces reveal a similar richness of possible shapes as the Co samples, for both the contact (Fig. 7) and the tunnel regime (Fig. 8). Several hysteretic jumps are observed in the low-field regime of the traces recorded for the atomic-size contacts. In the tunnel regime two types of traces are observed, one of which reveals a minimum of the resistance at $B=0 \mathrm{~T}$, while the other one depicts a hysteretic maximum. As for the Co samples a feature is observed at $|B|=1.8 \mathrm{~T}$, although in most traces with smaller amplitude than for the Co-only samples. Also the relative resistance changes are in the same order of magnitude as for the $\mathrm{Co}$ samples.

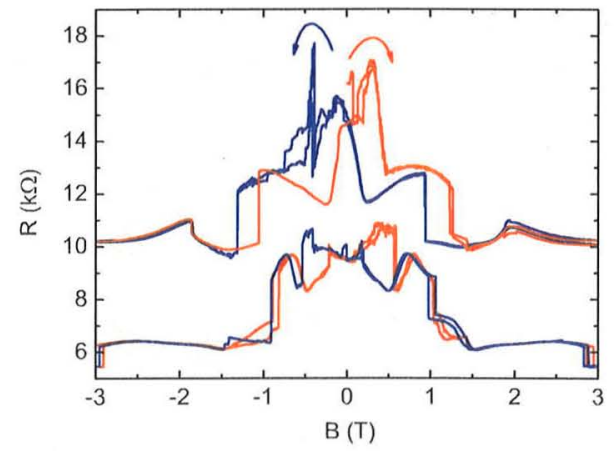

FIG. 7. (Color online) MR measurements of a Co-Au-Co break junction (sample No. 5) in the atomic-contact regime at $T<1.6 \mathrm{~K}$ in perpendicular field. Again, most of the traces reveal a hysteretic structure for $0<|B|<1 \mathrm{~T}$, and a reversible structure for higher fields as well as the structure around $|B|=1.8 \mathrm{~T}$. 

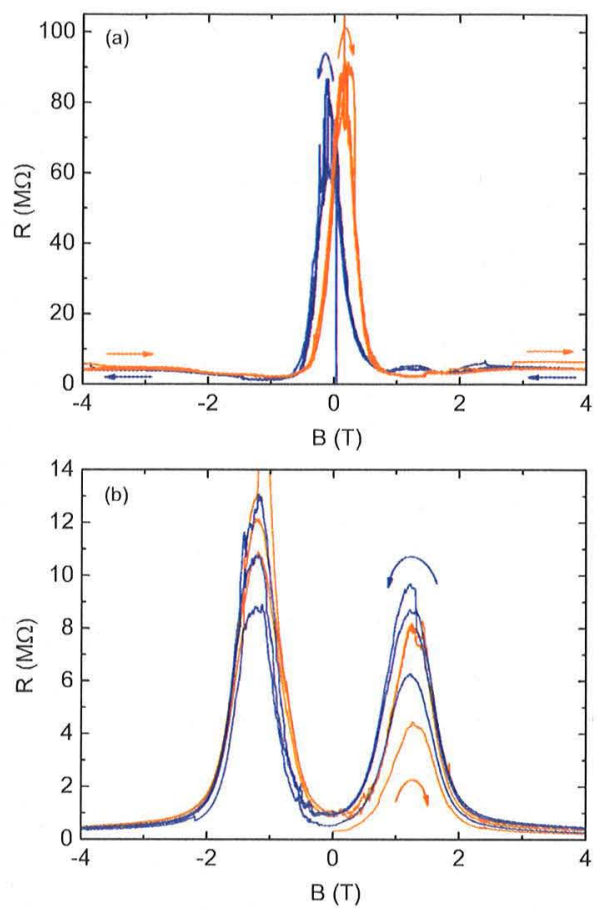

FIG. 8. (Color online) MR measurements of sample No. 5 in the tunnel regime for $T<1.6 \mathrm{~K}$ in perpendicular field. (a) For this contact a resistance minimum is observed at $B=0 \mathrm{~T}$. (b) A second type, which has been recorded in the same cooldown, reveals a hysteretic resistance maximum close to zero field. The resistance saturates above $|B|=3$ T.

For these samples we also performed measurements for field directions in the film plane (Fig. 9). The shape of the MR traces is much simpler with pronounced minima or maxima close to $50 \mathrm{mT}$, as found by Viret et al. ${ }^{19}$ and Shi $e t$ $a l^{23}$ The amplitude of the continuous changes is in the order of $50 \%$ and is interpreted as atomically enhanced anisotropic MR (AAMR) (Refs. 24 and 25) caused by an increased spinorbit coupling at the atomic level. Since the effect depends on the atomic configuration the enhancement factor of the saturation field varies from contact to contact. The same holds for the amplitude by which the resistance is changed by the AAMR effect. In addition, hysteretic jumps occur

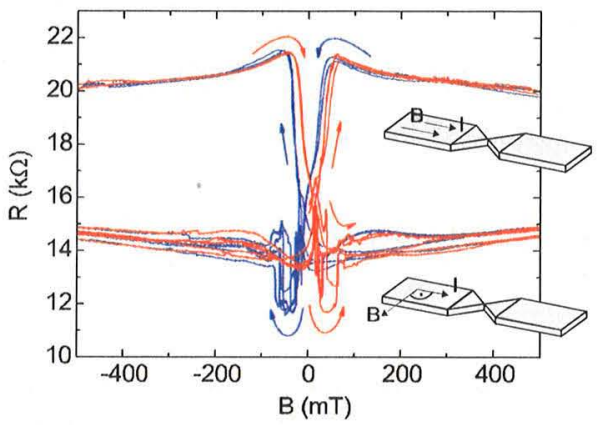

FIG. 9. (Color online) MR of a single-atom contact arranged from sample No. 5 for two field orientations (parallel and transverse to the current) in the sample plane.

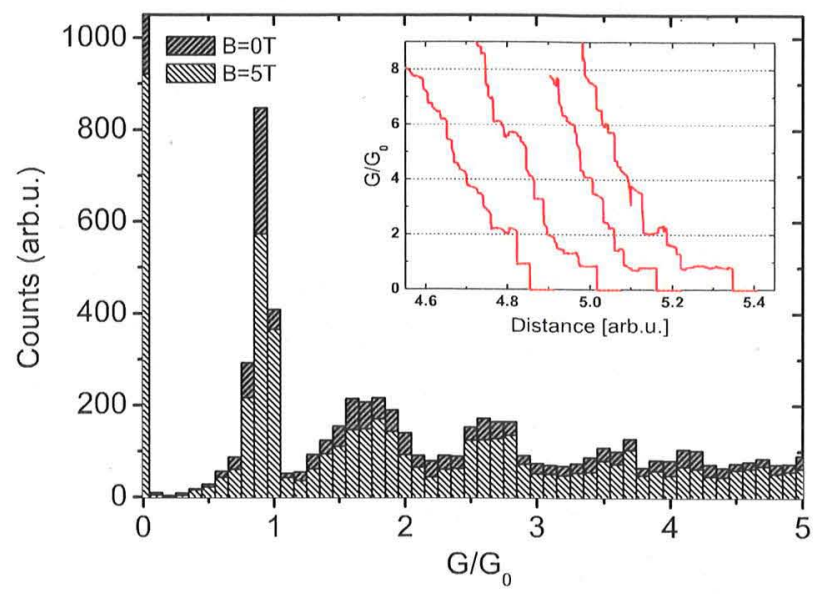

FIG. 10. (Color online) Main panel: opening histogram of a Au-Co-Au sample (No. 6) for $B=0$ T (right shaded, black bars) and $B=5 \mathrm{~T}$ (left shaded, white bars) recorded at $T=4.2 \mathrm{~K}$. Both reveal a peak just below $1 G_{0}$ which is typical for gold contacts. No clear peaks emerge at the value specific for Co single-atom contacts $\left(1.3 G_{0}\right)$. Moreover, there is no evidence for the dependence of the preferred conductance values on the external magnetic field. Inset: several opening curves of this sample. Rather long plateaus near $1 G_{0}$ are observed which are typical for single-atom contacts.

when applying the field transversely to the current direction. Possible origins for these jumps are discussed in Sec. IV.

\section{Au-Co-Au}

Complementary, the third class of specimen consists of gold leads, connected to a $300 \times 140 \mathrm{~nm}^{2}$ cobalt bridge [thickness $80 \mathrm{~nm}$, see Fig. 1(d)]. Although the large electrodes at a distance of about $200 \mathrm{~nm}$ consist of double layers of $\mathrm{Au}$ and $\mathrm{Co}$, the parts close to the contact are single layers made of Au. Only the triangular-shaped inner Au electrodes and the Co bridge are suspended. Thus, we assume that in first approximation, only the magnetic area of the bridge should affect the MR by its stray field, by the spin polarization of the conduction electrons, and finally by magnetostriction, although the latter contribution will be smaller than for the Co-only samples and the Co-Au-Co samples. Our first goal is again to determine which species of atoms forms the contact by recording conductance histograms (Fig. 10). A pronounced peak near $1 G_{0}$ and the absence of a peak at $1.3 G_{0}$ indicate that the junction breaks at one of the interfaces of the two materials but inside Au. This assumption is supported by the inspection of the sample in a scanning electron micrograph after the transport measurements [Fig. 1(d)]. The individual opening traces depict flat and long last plateaus which are typical for gold (inset of Fig. 10). Moreover, there is no evidence for the dependence of the preferred conductance values on the external magnetic field.

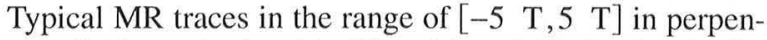
dicular field are depicted in Figs. 11 and 12. In the singleatom contact regime, we find a MR effect of the same order of magnitude as for the $\mathrm{Co}$ and the Co-Au-Co samples. The traces mostly show a double-peak structure in the contact 

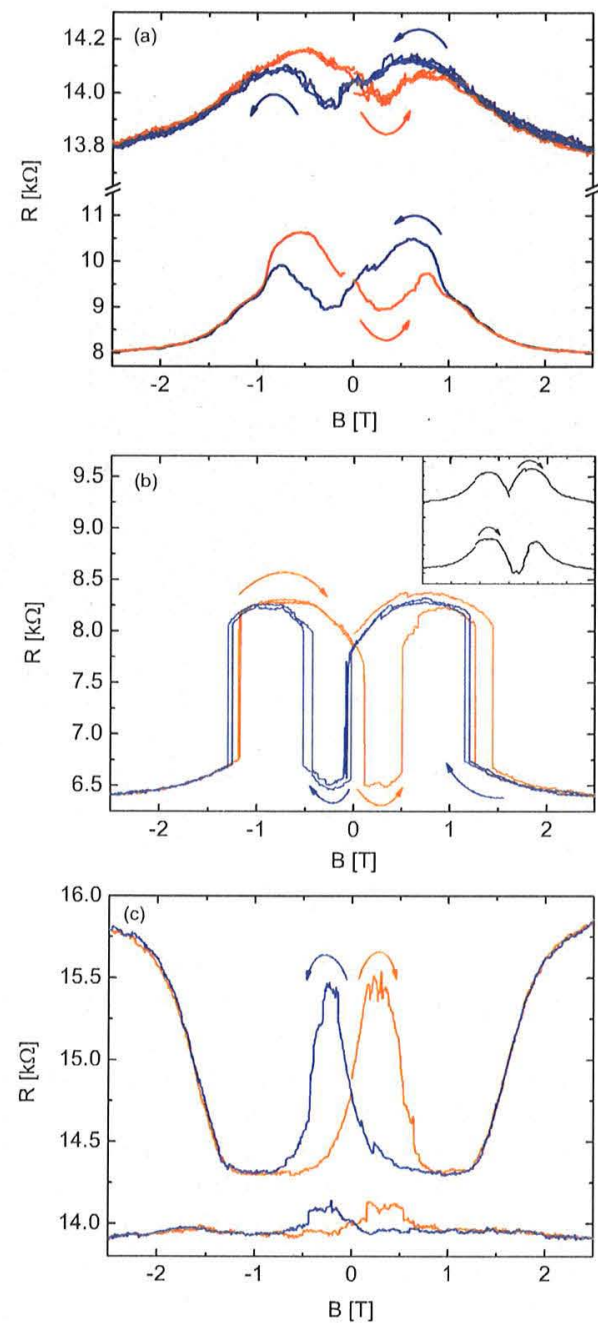

FIG. 11. (Color online) A selection of MR curves in the singleatom contact regime for sample No. 6 in perpendicular field: hysteretic maxima and minima are observed. In (b), superimposed conductance jumps are observed for magnetic fields lower than $\pm 1.5 \mathrm{~T}$. When subtracting these jumps, one obtains the usual double peak structure [inset of (b)], black curves.

regime as well as in the tunnel regime. The extremal values occur slightly shifted to smaller fields upon reversal of the sweep direction. In general, as shown in Fig. 11(a), the MR changes gradually, is reversible for $|B|>1 \mathrm{~T}$ and decreases at high fields. Some contacts also show superimposed discrete conductance jumps, as shown in Fig. 11(b). When subtracting these jumps [inset of Fig. 11(b)], one yields the usual structure. Finally, as shown in Fig. 11(c) hysteretic structures with gradual resistance changes are observed. Also for these $\mathrm{Au}-\mathrm{Co}-\mathrm{Au}$ samples we observe no saturation of the MR at high fields. However, no nonmonotonic behavior is observed above $|B| \approx 1 \mathrm{~T}$. Thus, the MR effects seem to occur on smaller field scale than for the other sample types. This is expected because due to its almost cubic shape the central Co bridge is expected to have a much smaller saturation field than the extended two-dimensional electrodes of the other samples.

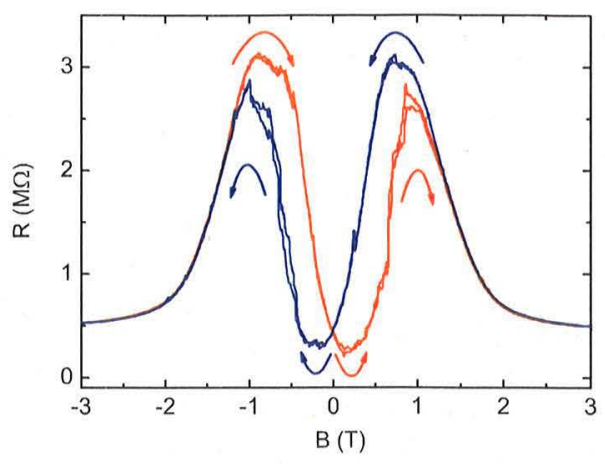

FIG. 12. (Color online) MR curve of sample No. 6 in the tunnel regime in perpendicular field. The traces show a double-peak structure with a small hysteresis in amplitude and in position. The minimum of the resistance occurs at $|B|=0.2 \mathrm{~T}$, the maximum near $\pm 1 \mathrm{~T}$. The maximum is shifted to smaller values when decreasing field amplitude. For higher fields the resistance decreases and saturates above $\pm 3 \mathrm{~T}$.

At variance to the other sample types ( $\mathrm{Co}, \mathrm{Co}-\mathrm{Au}-\mathrm{Co})$ we find here contacts with MRR values in the order of only $1-4 \%$. Interestingly these were found for conductance values of $0.90-0.96 G_{0}$ and thus correspond most likely to contacts within the Au (see Fig. 13). The lower curve in Fig. 11(c) shows an example of this low MRR. In contrast, the contact with $G \approx 1.3 G_{0}$ showed the highest MRR value in the order of 30\% [see lower curve in Fig. 11(a)].

\section{DISCUSSION}

The MR traces in perpendicular field of all samples show rich behavior and mostly continuous changes. In the contact regime, discrete resistance steps are observed for $|B|<1 \mathrm{~T}$. The observations are exceptional in the tunneling regime. The resistance changes persist above the saturation field of Co of $B=1.8$, the AAMR being the physical origin for this. ${ }^{25}$ Different contacts realized with the same sample give rise to

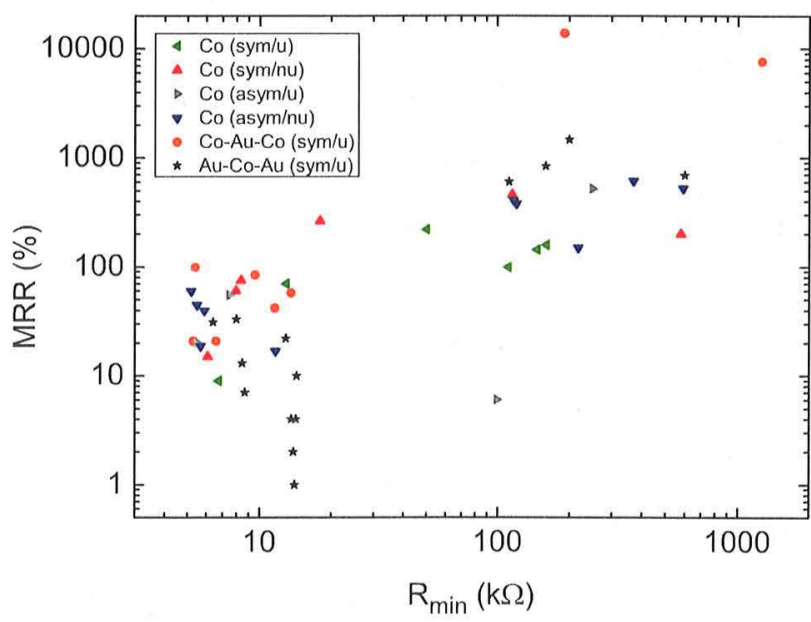

FIG. 13. (Color online) MRR values of selected contacts with all samples studied here: sym, asym, u, and nu. See also Table I. 
distinct shapes of the MR curves in accordance with the theoretical findings of Häfner et $a l .{ }^{11}$ The MRR values range from a few percent in the contact regime up to several hundred percent in the tunnel regime, summarized in Table I. Also the field at which the MR saturates varies from contact to contact for one and the same sample, as shown, e.g., in Fig. 4. In contrast to what has been reported before for $\mathrm{Ni}$ contacts, ${ }^{19}$ there is no particularly high value of the MRR around the single-atom contact.

Figure 13 shows a selection of the MRR as a function of the minimum resistance in the measured range. The MRR is smallest for the Au-Co-Au samples. Also, the traces recorded for these samples show less structure and no pronounced effects at or above the saturation field of $B=1.8 \mathrm{~T}$. From this finding we are able to estimate the influence of the correlations of the magnetization of the electrode to the MR. Interestingly, contacts with conductances close to $1 G_{0}$ reveal very small MRR values in consistency with the interpretation that these contacts are formed within the normal-metal part of the samples. Moreover, the MRR of nonsuspended samples is of comparable size as the one of suspended bridges. This observation is helpful for estimating the influence of magnetostriction. ${ }^{15}$

\section{A. Micromagnetic simulations}

The different shapes of the MR traces in the tunneling regime, cannot be explained in total by the AAMR effect, but rather by the micromagnetic structure of the electrodes forming the tunnel contact. For this purpose we performed threedimensional numerical simulations based on a macrospin mode ${ }^{26}$ using the material parameters of $\mathrm{Co}$. The spontaneous magnetization in our model is $M_{s}=1.43 \times 10^{6} \mathrm{~A} / \mathrm{m}$ and the exchange stiffness $A=2.6 \times 10^{11} \mathrm{~J} / \mathrm{m}$. The uniaxial anisotropy constant is $K=6.8 \times 10^{5} \mathrm{~J} / \mathrm{m}^{3}$ where the magnitude of the anisotropy is assumed to be constant but its direction is arbitrary in the film plane reflecting the granular structure of the cobalt. ${ }^{27}$ The value of the cell size $\Delta=7 \mathrm{~nm}$ corresponds to the grain size of our sample. Each cell contains one magnetic moment. Dipole-dipole interactions between grains are taken into account. For our simulation we use a Monte Carlo method with a heat-bath algorithm and singlespin-flip dynamics. ${ }^{28} \mathrm{~A}$ fast-Fourier transformation method is used in order to deal with the dipole-dipole interactions. ${ }^{29}$ We simulate a break junction of the size $896 \mathrm{~nm}$ $\times 448 \mathrm{~nm} \times 56 \mathrm{~nm}$, where the break is one cell size thick, applying a magnetic field $\mathbf{B}$ pointing out of plan.

We first carried out simulations for a structure without break. One finding of these simulations is that the remanent state shows a magnetization in plane with a multidomain vortex state in the inner electrodes. At the nanobridge region, a single-domain state is formed [Fig. 14(a)]. For the simulations with break in the center of the nanobridge, the same vortex structure is observed in the inner electrodes. Nevertheless, a multidomain vortex state with flux closure close to the break line is found [Fig. 14(b)]. Saturation in $z$ direction occurred at $|B| \approx 2 \mathrm{~T}$, slightly above the common value for cobalt $(1.8 \mathrm{~T})$.

We furthermore performed simulations for the exact sample geometry which we deduce from electron micro-

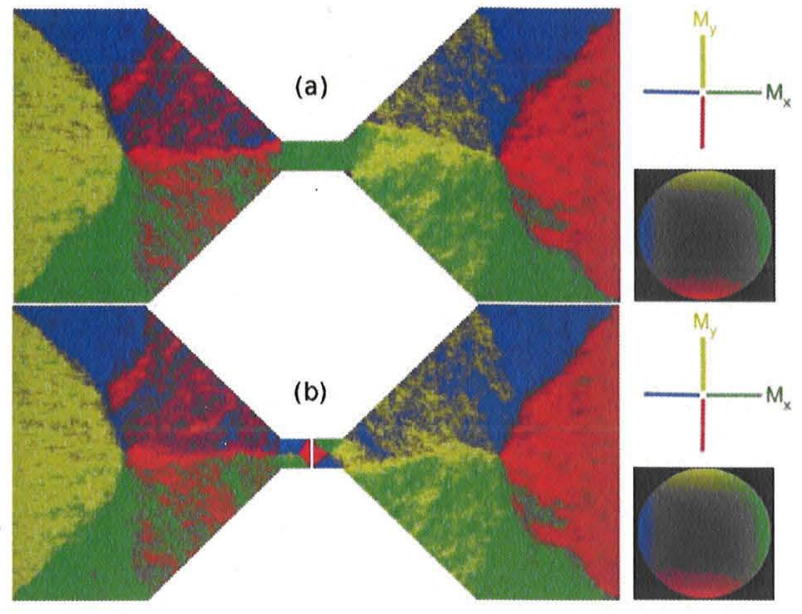

FIG. 14. (Color online) Simulation of the remanent magnetization state of the inner part for (a) a nonbroken and (b) a broken structure. The magnetization always shows a vortex structure in the inner electrodes. Without break (a), there is a single-domain state at the nanobridge region. In (b) the nanobridge region shows a vortex state with a flux closure close to the break line. The colored circle shows the direction of the magnetization.

graphs of the various samples. Comparing the domain structure of these slightly varying sample geometries we find that the exact shape of the magnetization distribution and, in particular, at the closest parts of the electrodes the relative alignment depends on the details of the geometry. More precisely, the remanent state also depends on the direction of saturation and is not necessarily symmetric with respect to field inversion because the two electrodes have slightly different shapes. Hence, for these remanent states nontrivial AMR behavior as well as nonmonotonous magnetostriction can be expected. Since magnetostriction depends on the domain configuration, every change in the magnetization state is accompanied by magnetostriction.

\section{B. Magnetostriction}

The notion of magnetostriction itself contains several aspects. The most commonly discussed is the length change caused by parallel orientation of the domains (Joule magnetostriction). ${ }^{30}$ For cobalt, the sample shortens upon alignment of the domains until it reaches its saturated magnetization state. Consequently, the maximum relative length change is limited to the anisotropic saturation coefficient $\lambda_{s}$. The size of $\lambda_{s}$ depends on the amount of fcc Co crystallites in the sample as well as on the substrate temperature and the deposition method. ${ }^{31}$ Since our samples are polycrystalline films with arbitrary orientation of the crystallites, we use the value $\lambda_{s}=-65 \times 10^{-6}$ measured by Chen et al. ${ }^{32}$ Moreover, the effect may have different sign and amplitude for magnetization along the easy and the hard axis, where a sign change in $\lambda_{s}$ as a function of the applied field can occur. There can also be a field-dependent contribution beyond the saturation field due to the volume dependence of the spontaneous magnetization (volume magnetostriction). ${ }^{30}$ 
To estimate the maximum contribution of magnetostriction to the MR, we use

$$
\Delta l / l=\frac{1}{2} \lambda_{s}\left(3 \cos ^{2} \theta-1\right)
$$

for the strain at magnetization angle $\theta$ in saturated magnetic field. ${ }^{15}$ Moreover, we assume that only the suspended parts of the bridges (length $u$ ) contribute to magnetostriction. Thus, in the tunnel regime length changes should give the most dramatic change in the resistance because of the exponential dependence on the tunneling gap. Considering Eq. (2), the maximum change in the tunneling gap at saturation would be $\Delta d=u \times \frac{3}{2} \lambda_{s} \approx 0.3-1 \AA$, depending on the sample structure. Using the usual formula for the distance variation in the tunneling current, we obtain

$$
R / R_{0}=\exp \left(\frac{2}{\hbar} \sqrt{2 m^{*} \Phi} \times \Delta d\right)=\exp (\beta \times \Delta d),
$$

where $\Phi=5 \mathrm{eV}$ is the work function ${ }^{33}$ and $m^{*}$ the effective mass of the conduction electrons. For cobalt, this value is not straightforward to determine, because both $s$ electrons and $d$ electrons can contribute to the transport of atomic-size contacts. ${ }^{10}$ We roughly determine the apparent $m^{*}$ of our Co nanobridges from the distance dependence in the tunnel regime when driving the motor without applied magnetic field and comparing it to the expected distance dependence given by the geometry of the breaking mechanism. ${ }^{34}$ We obtain $m^{* *} \approx m_{0}$ and therefore $\beta=2.3 \AA^{-1}$, which is in accordance with the findings in Ref. 10.

Our explanation for the qualitative behavior in the tunnel regime is the following: Since the remanent field state of the free-standing part of the bridge has a magnetization orientation in plane, a length change due to magnetostriction occurs until all domains are aligned in parallel along the bridge. This corresponds to a resistance increase because of a rising tunneling gap. At a certain field, the domains are tilted out of plane which closes the gap again and leads to a resistance decrease until saturation is reached. According to Eq. (3), at saturation, this estimated change in the gap distance could give rise to a resistance change in a factor of 2 for the $\mathrm{Au}$ $\mathrm{Co}-\mathrm{Au}$ samples and a factor of 10 for the $\mathrm{Co}$ and $\mathrm{Co}-\mathrm{Au}-\mathrm{Co}$ samples assuming that no "jump to contact" or "jump to tunneling" occurs, which could change the resistance considerably. Most of the observed resistance changes in the tunneling regime are in this order of magnitude. At higher fields the volume magnetostrictive effect comes into play. For Co it has the same sign as the orientational effect and should thus result in a monotonous resistance decrease up to the highest measurement fields.

The MR of the traces shown in Figs. 8(a) and 12 do indeed show examples in which the MR starts to increase when raising the field at $B=0$. However, there are even more examples in which it decreases. In particular, there are examples for which the sign of the tunnel MR varies from contact to contact in the same measurement run, i.e., without having demagnetized the sample. Moreover, there are many examples, e.g., the one shown in Fig. 4(b), where several sign changes are observed above the saturation field. Also the steepness of the MR decrease varies from contact to contact for the same sample [Fig. 4(a)]. From these findings we conclude that magnetostriction contributes to the MR of the tunnel contacts but it is not likely to account for the totality of the observations. For the atomic contacts the resistance change due to length changes in the electrodes is much smaller because the atomic bonds stabilize the geometry. Furthermore, the distance dependence of the resistance is weaker than exponential. The estimation above is thus an upper threshold for the resistance changes due to magnetostriction.

The third effect of magnetostriction is the development of an internal strain in the material when the outer edges are fixed such that a deformation is blocked (Villari effect). ${ }^{30}$ This strain may have an influence onto the resistance of atomic contacts by affecting the local band structure and is much more difficult to disentangle from other effects. A cosine-squared resistance behavior would also be expected for AMR, even without including the length change, which goes along with the reorientation of the domains. Deviations from this simple behavior have been reported for nanowires and nanosized constrictions. In particular, a strong enhancement of AMR in atomic-size structures has been described. $^{23,25}$ The accumulation of internal strain will eventually result in atomic rearrangements giving rise to sudden resistance jumps. We attribute the resistance jumps occurring at high magnetic fields to this effect.

\section{Hysteretic magnetoresistance}

Hysteretic effects are observed in all kinds of samples. However, the nature of the hysteresis varies. In some traces hysteretic switching, i.e., switching between two distinct resistance values in both sweep directions but at different field values is observed. This type occurs in all kinds of samples but not for all contacts which have been realized. E.g., the discrete steps in the order of $0.6-0.7 e^{2} / h$ in Fig. 11(b) resemble the ones reported by Shi et al. ${ }^{35}$ These steps have been explained as atomic motion induced by the applied magnetic field which can strongly affect the stability of atoms in the contact region. In contrast, Sokolov et al. ${ }^{36}$ attributed the conductance jumps in their experiments to BMR being caused by the opening and closing of discrete conductance channels in the contact. Herein, we propose that the observed conductance steps in our measurements are caused by field-induced atomic reconfigurations due to magnetostrictive forces.

The second type of hysteresis are abrupt resistance changes which only occur in one sweep direction, e.g., when enhancing the field amplitude. This is only observed for the $\mathrm{Co}$ and Co-Au-Co samples and at small field sizes. They do not occur for the Au-Co-Au samples. Thus, we attribute these steps to correlation effects between the two magnetic electrodes, e.g., the GMR or TMR effect. Both effects require sudden changes in the magnetization orientation at the interface between the ferromagnetic electrodes and a normal metal or insulating layer thinner than the spin-diffusion length. This is possible in both types of samples because a domain wall might be located close to the atomic contact. ${ }^{37}$ 
Furthermore sudden resistance jumps can be caused by magnetostrictive forces. In our samples we occasionally observe sudden resistance jumps at magnetic field amplitudes well above the saturation field (see, e.g., the feature around $3 \mathrm{~T}$ in the lower curve in Fig. 7). Since no sudden reorientations of magnetization is likely to occur at this fields, we attribute the jumps to magnetostrictive forces.

A third type of hysteresis goes along with continuous resistance changes with a functional dependence, which differs for both sweep directions. This effect is observed for all samples investigated, including the Au-Co-Au one which has only one magnetic part. The continuous hysteretic effects must be caused by a continuous change in a parameter. One possible explanation could be the deformation of a vortex state to a uniform magnetization direction or a monotonous tilt of the magnetization out of plane.

\section{High-field magnetoresistance}

Above the saturation field, the magnetization of the electrodes should be parallel to the applied field. Thus, all features in the MR at higher absolute field values must originate from either enhancements of the saturation field in the atomic contact due to the restricted geometry, volume magnetostriction, or the Villari effect. From the micromagnetic simulations we find that the magnetization of the nanocontact is tilted into the direction of the magnetic field at higher fields than the large electrodes. This is in accordance to what has been found for cobalt nanocontacts with magnetic field in plane.$^{38}$ However, above approximately $2.2 \mathrm{~T}$ the magnetization is completely saturated. The experimentally observed MR effects above this field have thus to be caused by a local enhancement (on a length scale smaller than the cell size of the simulations). This local enhancement must be caused by a variation in the local band structure at the atomic contact, i.e., the AAMR effect.

\section{CONCLUSIONS}

In conclusion, we presented a comprehensive study of the MR of magnetic atomic-size contacts realized by deforming free-standing metallic nanobridges. The MR in perpendicular magnetic field shows a rich behavior with relative resistance changes ranging from a few percent up to several thousand percent. By comparing the behavior of different sample lay- outs including the combination of magnetic with nonmagnetic parts and suspended versus nonsuspended nanobridges we were able to estimate the possible influence of magnetostriction.

We conclude that magnetostriction contributes to the observed MR, in particular, for tunnel contacts. Although the observed MRR values could be achieved by magnetostrictive effects, the shape of the MR traces cannot. In particular, the richness of the MR traces in the contact regime requires contributions of several effects. The effect giving rise to the strongest resistance changes are TMR as well as GMR for samples consisting of two magnetic electrodes. We estimate these contributions to be more than $50 \%$ of the total resistance change in the low-field regime of the atomic contacts. We argue that the continuous changes in the MR up to roughly $\pm 2 \mathrm{~T}$ are due to the reorientation of the magnetic domains in the electrodes, whereas all changes at higher fields are due to an enhancement of the saturation field in the constriction region. This enhancement is caused by strong spin-orbit scattering in atomic-size contacts and its effects have been called AAMR. For many contacts the saturation field must be enhanced by more than a factor of 2 because no saturation is observed up to our highest fields of $8 \mathrm{~T}$. The amplitude of this AAMR contribution to the MRR is hard to quantify because it strongly depends on the exact atomic arrangement. It gradually decreases from the single-atom contacts to the several-atom contacts because the orbital shape of the atomic wave functions become less important for bigger contacts. We estimate a contribution to the MRR value of roughly $10-20 \%$ (absolute) for the single-atom contacts. The observed discrete resistance jumps, in particular, those occurring at high fields can be attributed to atomic rearrangements triggered by magnetostrictive forces.

\section{ACKNOWLEDGMENTS}

We thank R. Arnold and M. Suty for their contributions to this work. We are indebted to C. Debuschewitz and V. Kunej for help and discussions about cryogenics issues. We profited a lot from discussions with J. C. Cuevas, M. Häfner, R. Hoffmann, C. Sürgers, and M. Viret. We gratefully acknowledge financial support from the DFG through SFB 513 and 767, from the Landesstiftung Baden-Württemberg and from the Alfried Krupp von Bohlen and Halbach foundation.
${ }^{1}$ H. D. Chopra and S. Z. Hua, Phys. Rev. B 66, 020403(R) (2002).

${ }^{2}$ N. García, M. Muñoz, G. G. Qian, H. Rohrer, I. G. Saveliev, and Y.-W. Zhao, Appl. Phys. Lett. 79, 4550 (2001).

${ }^{3}$ G. Tatara, Y.-W. Zhao, M. Muñoz, and N. García, Phys. Rev. Lett. 83, 2030 (1999).

${ }^{4}$ I. Mertig and P. Levy, in Theory of GMR in Spin Dependent Transport in Magnetic Nanostructures, edited by S. Maekawa (Taylor \& Francis, London, 2002).

${ }^{5}$ J. L. Costa-Krämer, Phys. Rev. B 55, R4875 (1997).
${ }^{6}$ C. Untiedt, D. M. T. Dekker, D. Djukic, and J. M. van Ruitenbeek, Phys. Rev. B 69, 081401(R) (2004).

${ }^{7}$ T. Ono, Y. Ooka, H. Miyajima, and Y. Otani, Appl. Phys. Lett. 75, 1622 (1999)

${ }^{8}$ F. Pérez-Willard, J. C. Cuevas, C. Sürgers, P. Pfundstein, J. Kopu, M. Eschrig, and H. v. Löhneysen, Phys. Rev. B 69, 140502(R) (2004).

${ }^{9}$ H. Suderow, M. Crespo, S. Vieira, M. Vila, M. Garcia Hernandez, A. de Andres, C. Prieto, C. Ocal, J. Martinez, and Y. Mukovskii, Physica E 18, 264 (2003). 


\section{MAGNETORESISTANCE OF ATOMIC-SIZE CONTACTS...}

${ }^{10}$ M. Häfner, J. K. Viljas, D. Frustaglia, F. Pauly, M. Dreher, P. Nielaba, and J. C. Cuevas, Phys. Rev. B 77, 104409 (2008).

${ }^{11}$ M. Häfner, J. K. Viljas, and J. C. Cuevas, Phys. Rev. B 79, 140410(R) (2009).

${ }^{12}$ Wen-C. Chiang, C. Ritz, K. Eid, R. Loloee, W. P. Pratt, and J. Bass, Phys. Rev. B 69, 184405 (2004).

${ }^{13}$ C. J. Muller, J. M. van Ruitenbeek, and L. J. de Jongh, Physica C 191, 485 (1992).

${ }^{14}$ J. M. van Ruitenbeek, A. Alvarez, I. Piñeyro, C. Grahmann, P. Joyez, M. H. Devoret, D. Esteve, and C. Urbina, Rev. Sci. Instrum. 67, 108 (1996)

${ }^{15}$ M. Gabureac, M. Viret, F. Ott, and C. Fermon, Phys. Rev. B 69 , 100401(R) (2004).

${ }^{16}$ Z. K. Keane, L. H. Yu, and D. Natelson, Appl. Phys. Lett. 88, 062514 (2006)

${ }^{17}$ B. Leven and G. Dumpich, Phys. Rev. B 71, 064411 (2005).

${ }^{18}$ H.-X. Wei, T.-X. Wang, E. Clifford, R. M. Langford, X.-F. Han, and J. M. D. Coey, J. Appl. Phys. 99, 08C512 (2006).

${ }^{19}$ M. Viret, S. Berger, M. Gabureac, F. Ott, D. Olligs, I. Petej, J. F. Gregg, C. Fermon, G. Francinet, and G. Le Goff, Phys. Rev. B 66, 220401(R) (2002).

${ }^{20}$ T. McGuire and R. Potter, IEEE Trans. Magn. 11, 1018 (1975).

${ }^{21}$ J. F. Gregg, W. Allen, K. Ounadjela, M. Viret, M. Hehn, S. M. Thompson, and J. M. D. Coey, Phys. Rev. Lett. 77, 1580 (1996).

${ }^{22}$ N. Agraït, A. Levy Yeyati, and J. M. van Ruitenbeek, Phys. Rep. 377, 81 (2003).

${ }^{23}$ S.-F. Shi, K. I. Bolotin, F. Kuemmeth, and D. C. Ralph, Phys.
Rev, B 76, 184438 (2007)

${ }^{24} \mathrm{G}$. Autès, C. Barreteau, M. Desjonquères, D. Spanjaard, and M. Viret, EPL 83, 17010 (2008).

${ }^{25}$ M. Viret, M. Gabureac, F. Ott, C. Fermon, C. Barreteau, G. Autes, and R. Guirado-Lopez, Eur. Phys. J. B 51, 1 (2006).

${ }^{26}$ B. Hausmanns, T. P. Krome, G. Dumpich, E. F. Wassermann, D. Hinzke, U. Nowak, and K. D. Usadel, J. Magn. Magn. Mater. 240, 297 (2002).

${ }^{27}$ M. Brands, R. Wieser, C. Hassel, D. Hinzke, and G. Dumpich, Phys. Rev, B 74, 174411 (2006).

${ }^{28}$ U. Nowak, Annu. Rev. Comput. Phys. IX, 105 (2001).

${ }^{29}$ D. Hinzke and U. Nowak, J. Magn. Magn. Mater. 221, 365 (2000).

${ }^{30}$ E. W. Lee, Rep. Prog. Phys. 18, 184 (1955).

${ }^{31}$ E. Klokholm and J. Aboaf, J. Appl. Phys. 53, 2661 (1982).

${ }^{32}$ Y. Chen, B. K. Kriegermeier-Sutton, J. E. Snyder, K. W. Dennis, R. W. McCallum, and D. C. Jiles, J. Magn. Magn. Mater. 236, 131 (2001).

${ }^{33}$ H. B. Michaelson, J. Appl. Phys. 48, 4729 (1977).

${ }^{34}$ S. A. G. Vrouwe, E. van der Giessen, S. J. van der Molen, D. Dulic, M. L. Trouwborst, and B. J. van Wees, Phys. Rev. B 71, 035313 (2005).

${ }^{35}$ S.-F. Shi and D. C. Ralph, Nat. Nanotechnol. 2, 522 (2007).

${ }^{36}$ A. Sokolov, C. Zhang, E. Tsymbal, J. Redepenning, and B. Doudin, Nat. Nanotechnol. 2, 171 (2007).

${ }^{37}$ P. Bruno, Phys. Rev. Lett. 83, 2425 (1999).

${ }^{38}$ G. Sarau and C. M. Schneider, J. Appl. Phys. 102, 083907 (2007). 